\title{
A Method in Smoothing Fluctuation of Wind Power with Wavelet Threshold Filtering and Mean-value Intervals by HESS
}

\author{
SUN Bingying ${ }^{1, ~ a, ~ L I U ~ Z o n g q i ~}{ }^{1, b}$, YANG Shuili ${ }^{2, c}$ and ZHANG Boyue ${ }^{1}$
}

\author{
${ }^{1}$ School of Electrical and Electronic Engineering, North China Electric Power University, Changping \\ District, Beijing 102206, China \\ ${ }^{2}$ China Electric Power Research Institute, Haidian District, Beijing 100192, China \\ asunby2015@163.com, 'Izq@ncepu.edu.cn, cyangsl@epri.sgcc.com.cn
}

\begin{abstract}
Keywords: Wind power fluctuation, hybrid energy storage system, smooth method, wavelet threshold filtering, mean-value interval

Abstract. Due to the inherent fluctuation nature of wind power, voltage control and frequency regulation in China's power grid become more intricate as the wind power penetration level increasing. Provided with traits of comprehensive response and bidirectional output, wide concern has been arisen by the hybrid energy storage system (HESS), while how to design a smooth strategy is vital to achieve great effect. In order to conform the fluctuation of the wind power to China's technical standards, based on a HESS composed of electrical double layer capacitors (EDLCs) and battery, this paper proposes a strategy utilizing wavelet threshold filtering and mean-value interval as first and second smooth step, to flat the high and low frequency components of wind power fluctuations respectively. Taking a wind farm as an illustration, the validity of this strategy is verified by the calculation and analysis.
\end{abstract}

\section{Introduction}

With larger-scale wind farms integrated into power grid in northern China, owing to the weak capability of wind turbines in outputs control [1], regional grid can scarcely acquire prompt and active supports for frequency and voltage from them. Meanwhile, primary frequency control in whole grid, as well as the rotating inertia of the sending and receiving ends has been dampened with the declined proportion of conventional units [2]. Besides, the existing AC power grid's deficiency in bearing load transfer results in its difficulty to respond effectively to surging power flow, which would causes voltage instability. However, current grid technology is unable to solve all issues completely, consequently forced to abandon wind power. Hence how to smooth fluctuations effectively under the premise of safety and stability becomes the pivot to develop clean energy.

The direct method that adjusting the pitch angle by wind turbine to smooth is inadequate and lowers the utilization of wind [3]. Yet energy storage devices, with advantages of fast-response and bidirectional flexibly control of power, can regulate the outputs of a wind farm immediately [4]. Currently allocating wind farms with energy storage is a valid method to soften fluctuant impacts on the grid [5]. Moreover, combining two or more sorts of energy storage devices as a HESS, which exhibits comprehensive characteristics, performs better in smoothing comparing to single one [6]. Relevant researches on smooth strategy have been carried out by scholars, wind power signal is decomposed into high and low frequency components via wavelet transform in [7], and HESS absorbs high and sub-high frequency components respectively. Yet low-frequency but large-power components get no smooth and without a target. To maximize the benefits of wind-storage system, an optimal control method is proposed in [8] to inhibit the low ramping rate components.

High and low frequency components of fluctuations influence relatively the primary frequency regulation and AGC of power grid [9]. Merely stabilize the former but ignore the latter will also overburden AGC work. Thus, considering distinct response-rate of EDLCs and battery, with the smoothing target given by China's technical rule, this paper proposes a method based on wavelet threshold filtering and mean-value intervals to restrain fast fluctuation by EDLCs and slow fluctuation by battery. Besides, this paper analyzes the validity of the method and accordingly acquires power reference value of EDLCs and battery required by flattened outputs. 
This paper starts by giving the connection structure of a wind-HESS system to power grid in Section II, the proposed smooth strategy is defined in Section III. The paper then continue by analyzing an illustration with the method in Section IV and by making conclusions in Section V.

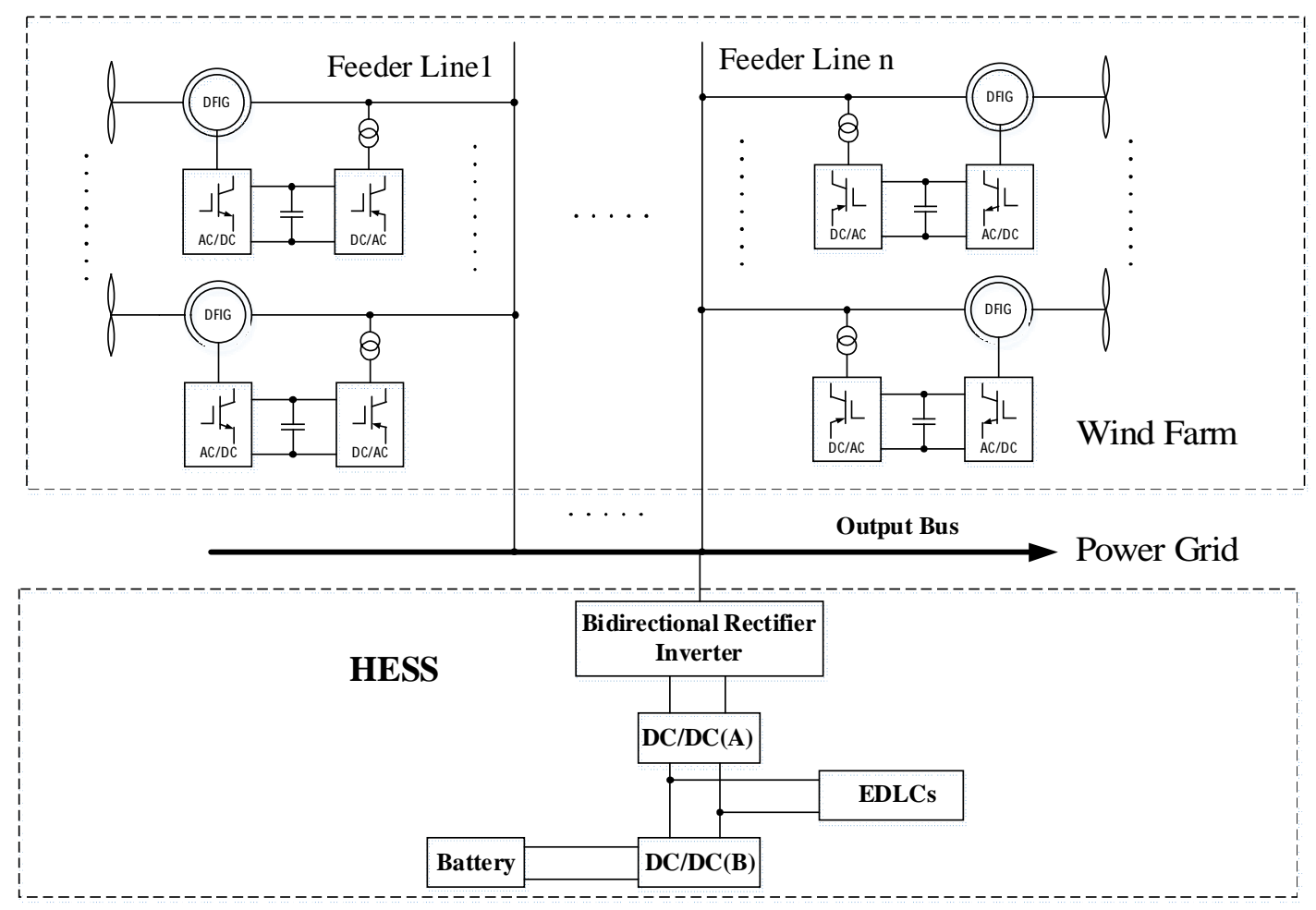

Fig.1 Schematic diagram of power system containing Wind Power and HESS

\section{Wind-HESS system}

Doubly-fed Induction Generators (DFIG) are integrated via several feeder lines into a bus, where a HESS is arranged. The structure is shown in Fig.1. The HESS includes a battery energy storage system (BESS) and EDLCs, which are connected in parallel in a dual DC/DC structure. The high-voltage side of DC/DC (B) is paralleled to the low-voltage side of DC/DC (A), and then the another side of (A) as the DC-voltage outlet, is connected to a rectifier/inverter set in the bus integrated into the power grid.

Considering battery with high energy-density [10] and EDLCs with high power-density [11], distinct advantages of both should be utilized to design the smooth strategy to attain high efficiency.

\section{Smooth strategy}

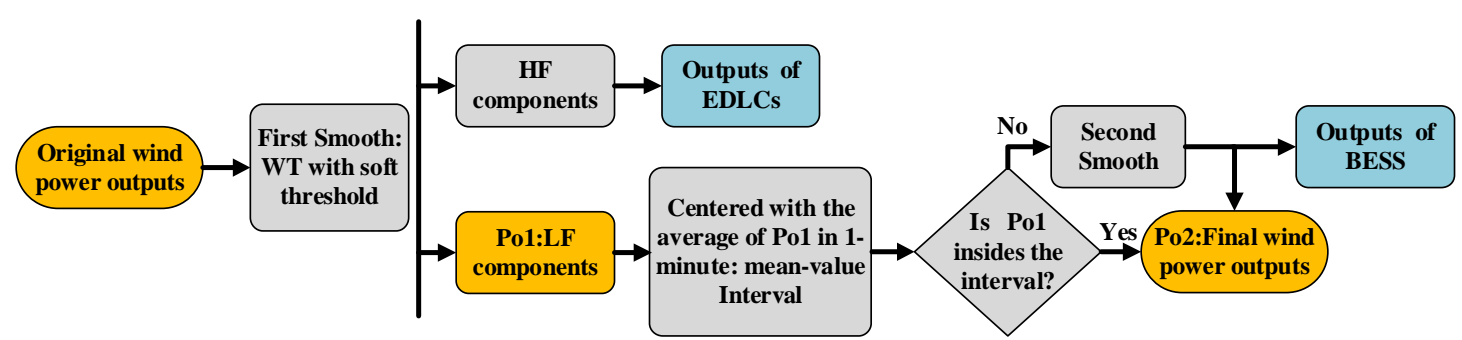

Fig.2 Flow chart of Smoothing Method of HESS

The strategy is designed as follow: firstly, wind power signal is discomposed into approximation and details by wavelet transform with soft threshold. Secondly, details as high-frequency components are absorbed by faster-responsive EDLCs and $P_{o 1}$ is obtained as first output. Thirdly, based on the mean-value of $P_{o 1}$, second smoothing interval is built, and BESS is utilized to absorb 
the extra parts outsides the interval, then the final signal is attained as $P_{o 2}$.

A. Smooth target

China's national technical standards document GBT_19963-2011 "technical rule for connecting wind farm to power system" was promulgated in 2011, and the wind farm's maximum limit of active power's variation in 1 minute has been given in section 5.1.3, as shown in Table 1.

Table. 1 maximum limit of active power's variation in 1 minute (MW)

\begin{tabular}{cc}
\hline Wind farm's Capacity & Limit \\
\hline$<30$ & 3 \\
$30-150$ & Capacity/10 \\
$>150$ & 15 \\
\hline
\end{tabular}

The target of strategy is to decline the variations of final outputs to the limit. An index is also given to describe the smoothing effect and defined as:

$$
\xi=\frac{\alpha-\beta}{\alpha} \times 100 \%
$$

Here $\alpha$ and $\beta$ refer to the wind farm's maximal variation before and after the smooth respectively, $\xi$ is known as the fluctuation reduction rate.

B. First smooth: wavelet threshold filtering

The principle of wavelet transform is:

$$
x(t)=\sum_{j} \sum_{k} w(j, k) \varphi_{j, k}(t) .
$$

Here $x(t)$ is the original signal, $w(j, k)$ are the wavelet coefficients to be found using transform wavelets $\varphi_{j, k}(t) . \varphi_{j, k}(t)$ can be acquired from mother wavelet $\varphi(t)$ as:

$$
\varphi_{j, k}(t)=a^{-j / 2} \varphi\left(\frac{t-k b a^{j}}{a^{j}}\right) \text {. }
$$

Here $a$ and $b$ are usually 2 and 1 respectively. And $w(j, k)$ can be got by the inner product of $x(t)$ and $\varphi_{j, k}(t)$ as:

$$
w(j, k)=<x(t), \varphi_{j, k}(t)>.
$$

Taking the rated power of EDLCs into account, heavy cost might be caused as absorbing all of sharp-changing parts in first smooth, and also unnecessary. Therefore, a soft threshold is introduced to sift all the wavelet coefficients obtained above, among of which is larger than the threshold could be set as zero while the others remain unchanged. By this method, most of the high-frequency components are filtered out, only several high-frequency components with large power are preserved to be absorbed in second smooth by the battery. Filtering operation is as follows:

a. Carry on 5-layer wavelet decomposition by Mallat algorithm.

Each layer resembles to the signal going through a low and a high pass filter, and the low-frequency signal got from last layer can be decomposed again as:

$$
A_{j} x=A_{j+1} x+D_{j+1} x
$$

$A_{j} x$ are the images under resolution of $2^{-j}$, referred as approximation and $D_{j} x$ as details:

$$
\begin{aligned}
& A_{j} x=\sum_{k} c(j, k) \phi_{j, k}(t)=\sum_{k}<x(t), \phi_{j, k}(t)>\phi_{j, k}(t), \\
& D_{j} x=\sum_{k} d(j, k) \psi_{j, k}(t)=\sum_{k}<x(t), \psi_{j, k}(t)>\psi_{j, k}(t) .
\end{aligned}
$$

Here $c(j, k)$ are scale expansion coefficients and $d(j, k)$ are detail coefficients, which can be used to attain approximation and details respectively, while $\square j_{j, k}(t)$ and $\psi_{j, k}(t)$ are relatively the scale 
function of approximate and detailed parts. Finally 5-layer wavelet decomposition of original wind power is carried out as:

$$
x=a_{5}+d_{5}+d_{4}+d_{3}+d_{2}+d_{1} .
$$

Here $d_{i}$ are detailed parts and mainly contain high-frequency components, $i=1,2,3,4,5$. And $a_{5}$ mainly refers low-frequency components.

b. Select a threshold function to process wavelet coefficients.

With a soft threshold function, the new wavelet coefficients are generated as:

$$
w_{\text {new }}(j, k)=\left\{\begin{array}{l}
\operatorname{sign}(w(j, k))(|w(j, k)|-\lambda),|w(j, k)| \geq \lambda \\
0,|w(j, k)|<\lambda
\end{array} .\right.
$$

Here $c(j, k)$ and $d(j, k)$ are both included in $w(j, k), \lambda$ is the threshold found by:

$$
\lambda=\sigma^{2} \lg (M)
$$

$M$ refers to the length of input signal, $\sigma$ can be found by the module value of $w(j, k)$ as an estimate to the noise contained in the signal:

$$
\sigma=\frac{\text { median }(|w(j, k)|)}{0.6745} .
$$

c. Reconstruct outputs using the wavelet coefficients.

The outputs of wind power after wavelet threshold filtering is illustrated as:

$$
P_{o 1}(t)=\sum_{k} c_{n e w}(3, k) \phi_{3, k}(t)+\sum_{j=1}^{3} \sum_{k} d_{n e w}(j, k) \psi_{j, k}(t) .
$$

d. Get the outputs of EDLCs

The smooth quantity $P_{\text {flat } 1}$ equals to the difference between $x(t)$ and $P_{o 1}$ :

$$
P_{\text {flat } 1}=x(t)-P_{o 1}(t) \text {. }
$$

Then the expected outputs of EDLCs is illustrated as:

$$
P_{\text {edlcs }}=-P_{\text {flat } 1} \text {. }
$$

C. Second Smooth: mean-value intervals

Mere filter is not sufficient to achieve the smoothing target, while low-frequency components are also supposed to be processed in the second smooth dominated by the battery, which possesses the high energy-density and large throughput. The interval is centered with the mean-value $P_{a v g}$ of $P_{o 1}$ in 1 minute. Provided the number of sampling points is $\mathrm{n}$ per minute, $P_{\text {avg }}$ in $\tau$-th minute is found as:

$$
\begin{aligned}
& P_{\text {avg }}(\tau)=\sum_{i=1}^{n} P_{o 1}(n(\tau-1)+i) . \\
& P_{\text {flat } 2}=-\left\{\begin{array}{l}
\left(P_{o 1}-P_{\text {avg }}\right)-5 \% P_{\text {avg }}, \frac{P_{o 1}-P_{\text {avg }}}{P_{\text {avg }}}>5 \\
\left(P_{o 1}-P_{\text {avg }}\right)+5 \% P_{\text {avg }}, \frac{P_{o 1}-P_{\text {avg }}}{P_{\text {avg }}}<-5 \% \\
0, \text { else }
\end{array}\right.
\end{aligned}
$$

Set the neighborhood as $\left[(1 \pm 5 \%) P_{a v g}\right]$, and the flatten quantity can be obtained as equation (16). 


\section{Case analysis}

The case utilizes outputs data on Jan.6, 2010 of a wind farm located in northern China. Its installed capacity is $100 \mathrm{MW}$, with the sampling interval of $12 \mathrm{~s}$ and 7200 points are obtained.

A. First smooth

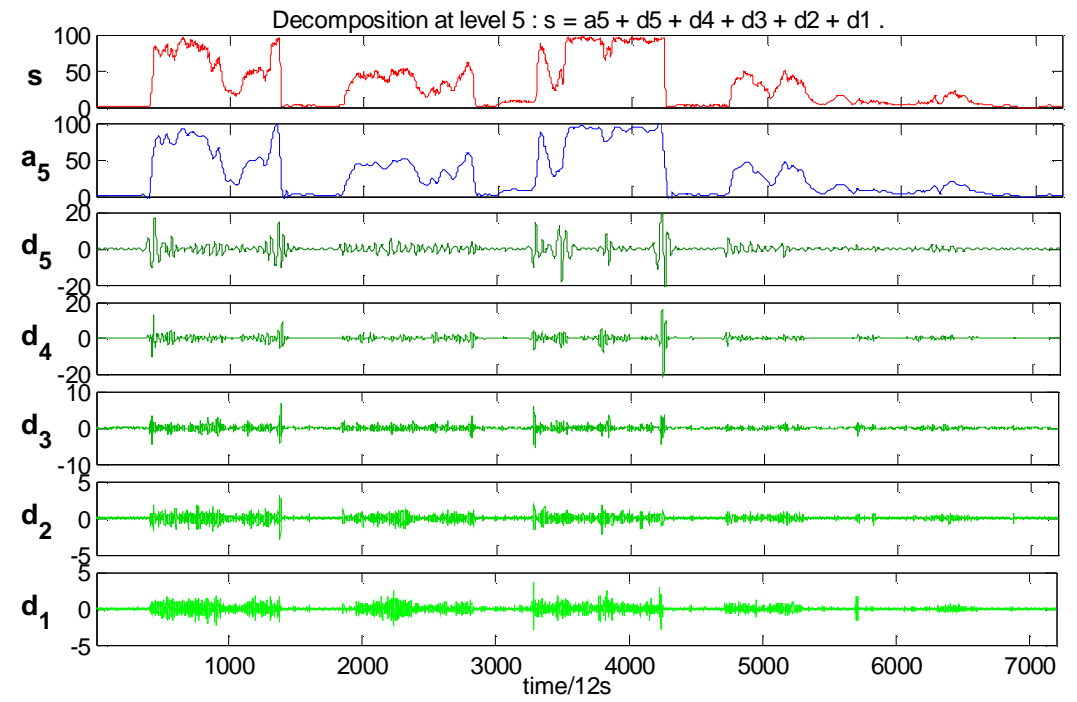

Fig.3 db4 Wavelet Transformation to Original Wind Power Signal in 5 Levels
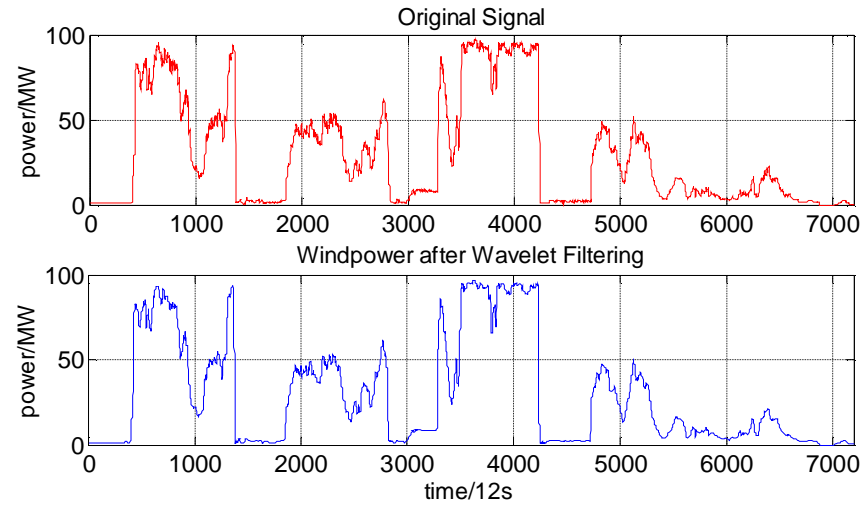

Fig.4 Outputs before and after First Smooth
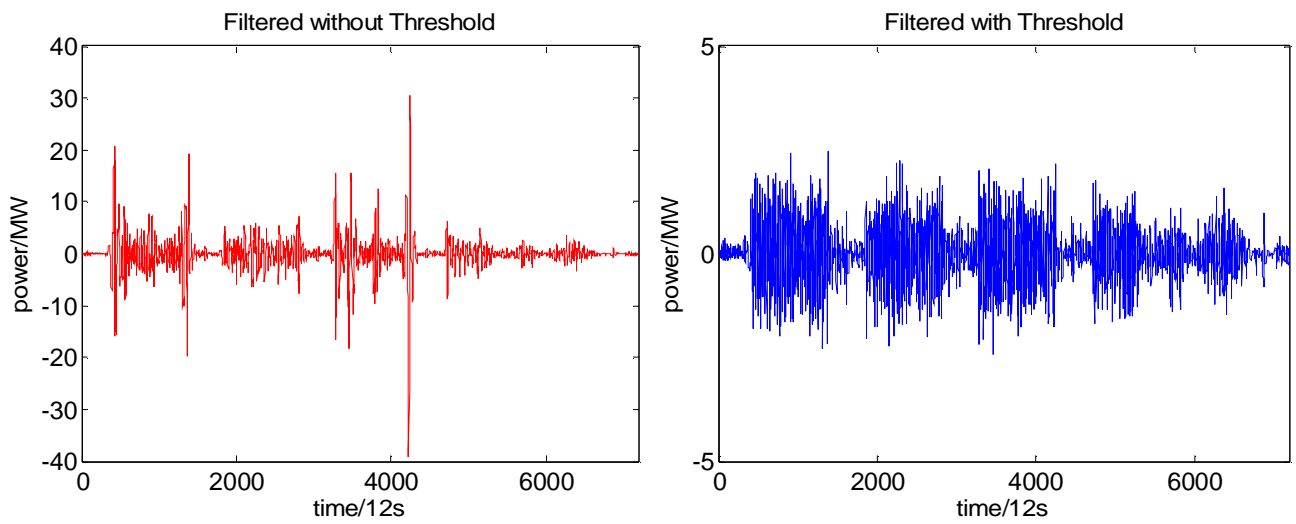

Fig.5 Outputs of EDLCs during First Smooth before and after Threshold Set

a. 5-layer wavelet decomposition

The db4 wavelet is used to decompose the original outputs, detailed and approximate parts are shown in Fig.3.

b. Threshold process and reconstruction

A Soft-threshold function is used to handle wavelet coefficients got in $a$. Comparison between 
fluctuate signals before and after first smooth is as Fig.4.

c. Outputs of EDLCs

The outputs of the EDLCs before and after the introduction of threshold is compared in Fig.5.

B. Second smooth

With mean-value power in 1 minute computed by $P_{o 1}$, low-frequency fluctuations to be smoothed can be put out utilizing the function $P_{\text {flat }}$ illustrated as equation (15). Final outputs $P_{o 2}$ is shown in Fig. 6 and the output curve of battery is shown in Fig.7.
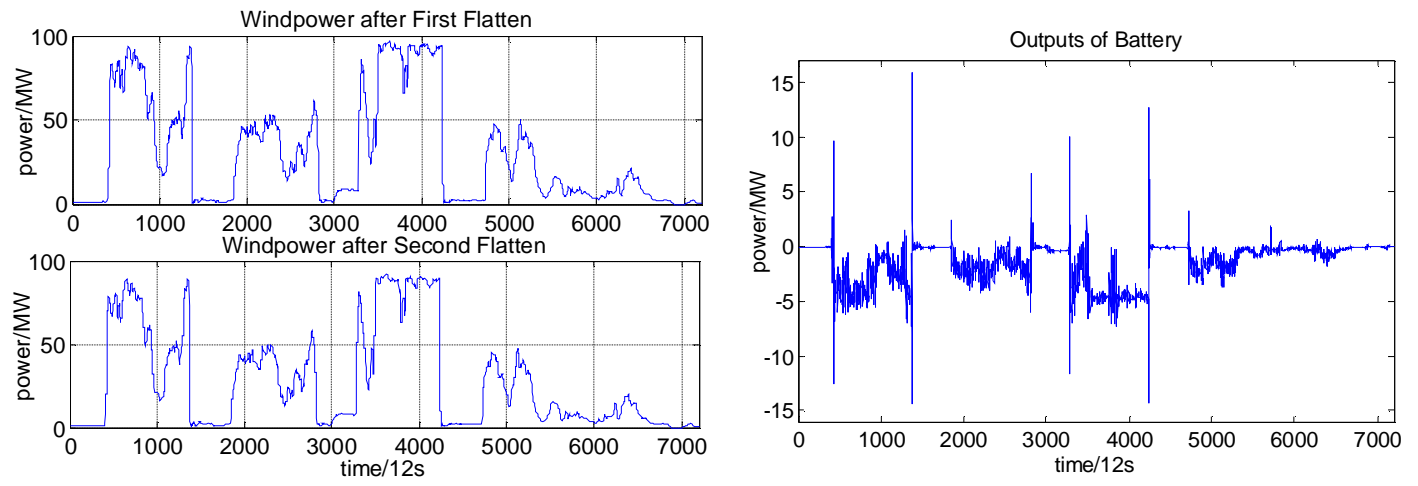

Fig.6 Outputs before and after Second Smooth

Fig.7 Outputs of Battery during Second Smooth

\section{Effects analysis}

After first and second smooth, the distribution of variation's maximum of can be attained from signal $P_{o 1}$ and $P_{o 1}$, as Fig. 8 shows. All relative data is listed in Table 2.

From Fig.8, maximal variation after first and second smooth both decrease, and the reduction rate $\xi$ is worked out as $3.558 \%$ and $75.755 \%$ respectively.

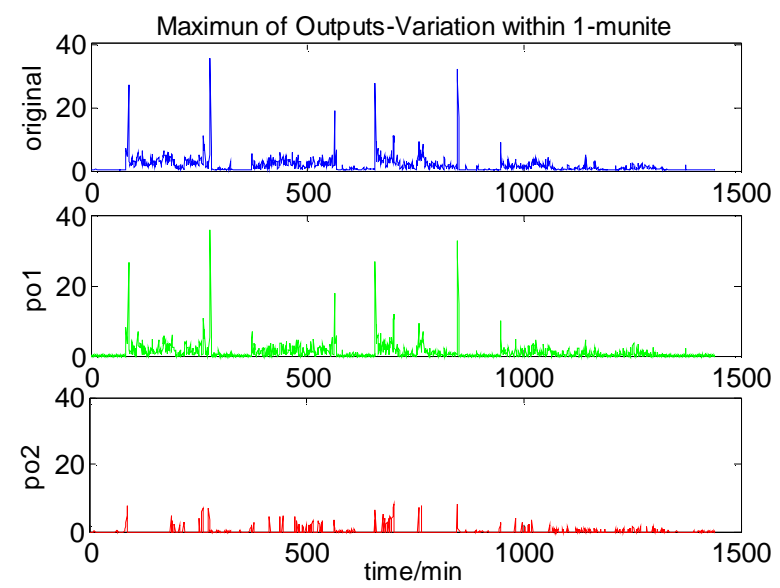

Fig.8 Maximum of Outputs-Variation within 1-minute before and after Smooth

From Table.2, final maximum fell to $8.2353 \mathrm{MW}$ and controlled below $10 \mathrm{MW}$, the $10 \%$ of installed capacity of the wind farm, restricted by the technique rule.

Table. 2 maximum of variation in 1 minute before and after smooth (MW)

\begin{tabular}{cccc}
\hline & Original & $P_{o 1}$ & $P_{o 2}$ \\
\hline Max & 35.2198 & 33.9667 & 8.2353 \\
\hline
\end{tabular}

D. Power requirements of energy storage devices

Table. 3 maximal output of EDLCs and BESS (MW)

\begin{tabular}{cccc}
\hline & EDLCs(with threshold) & EDLCs(without threshold) & BESS \\
\hline Export & 2.4624 & 30.6218 & 15.8487 \\
Absorb & 2.4426 & 39.129 & 14.3839 \\
\hline
\end{tabular}

Maximal output of EDLCs and BESS are listed in Table.3. The maximum of EDLCs declined to 2.4624MW after introducing a threshold function. As a reference, EDLCs' rated power should be 
set larger than 2.2624MW and BESS's should be greater than 15.8487MW.

\section{conclusion}

This paper proposes a strategy, based on comprehensive traits of HESS and variation limits given by technique rule, to smooth the fluctuations of wind power. The main conclusions are as follows:

(1) The wavelet threshold filtering and mean-value flat methods are proposed;

(2) By a simulation experiment based on the data of a wind farm, the effectiveness of the strategy is validated as the variation in 1 minute declined by $76.61 \%$, which is allowed by technical rule to integrate into the grid. In given scenario, the rated power allocation of EDLCs and BESS should be no less than 2.2624MW and 15.8487MW respectively.

\section{Acknowledgements}

This work was financially supported by Science and Technology Project (KY-SG-2016-204-JLDKY) of State Grid Corporation of China.

\section{References}

[1] E.A. DeMeo, W. Grant, and M. J. Schuerger, "Wind plant integration, costs, status, and issues," IEEE Power \& Energy Magazine, pp. 38-46, Dec. 2005.

[2] Hadi Banakar, Changling Luo,Boon Teck Ooi, et al. Impacts of Wind Power Minute-to-Minute Variations on Power System Operation [J]. IEEE Transactions on Power Systems, Vol.23, No.1, February, 2008

[3] Senjyu T, Sakamoto R. Output power leveling of wind turbine generator for all operating regions by pitch angle control [J]. IEEE Trans on Energy Conversion, 2006, 21(2): 467-475.

[4] Olivia Leitermann, James L.Kirtley. Energy storage for use in load frequency control [J]. IEEE, 2010, 292-296.

[5] Barton J, Infield D. Energy storage and its use with intermittent renewable energy [J]. IEEE Transactions on Energy Conversion, 2004, 9(2): 441-448 .

[6] ZHANG Guoju, TANG Xisheng, and QI Zhiping. Design of a hybrid energy storage system on leveling of fluctuation power outputs of intermittent sources [J]. Automation of Electric Power Systems. Vol.35 No.20, Oct.25, 2011

[7] HAN Xiaojuan, CHEN Yueyan, and ZHANG Hao. Application of Hybrid Energy Storage Technology Based on Wavelet Packet Decomposition in Smoothing the Fluctuations of Wind Power [J]. Proceedings of the CSEE. Vol.33. No.8, July.5, 2013

[8] WANG Ying, ZHANG Kaifeng, FU Jiayu, et.al. Optimization control method of wind/storage system for suppressing wind power ramp rate $[\mathrm{J}]$. Automation of Electric Power Systems. Vol.13. No.17, Oct.25, 2011

[9] Yuri V. Makarov, Clyde Loutan, Jian Ma1, et al. Impacts of Wind Generation on Regulation and Load Following Requirements of California Power Systems [M]. IEEE, 2008, 1-6.

[10]Dogger.J.D, Roossien B, Nieuwenhout.F.D. Characterization of li-ion batteries for intelligent management of distributed grid-connected storage [J]. IEEE Transactions on Energy Conversion, 26(1):256-263

[11] Mikio Fukuhara,Kazuyuki Sugawara. Electric charging/discharging characteristics of super capacitor, using de-alloying and anodic oxidized Ti-Ni-Si amorphous alloy ribbons [J]. Nanoscale Research Letters, 2014 